\title{
Galileo Signals Acquisition Using Enhanced Subcarrier Elimination Conversion and Faster Processing
}

\author{
Ali Albu-Rghaif, Ihsan A. Lami, Maher Al-Aboodi, Patrick Van Torre, Hendrik Rogier
}

\begin{abstract}
To solve multipath and to enhance the localisation accuracy in a harsh environment, BOC modulation has been adopted in modern GNSS transmission, such as GPS-M-code and Galileo-OS-code signals. The designers of the BOC technique have pointed out that the correlation function becomes ambiguous when the received signal is correlated with the reference BOC signal at code phase resolutions of 0.5 Chip. This has motivated many contributions to resolving this ambiguity, for example, by processing each side of the BOC lobes as a BPSK signal. Our literature survey concluded that solutions claiming to have mitigated this ambiguity actually have resulted in a more complex receiver implementation. The Enhanced Subcarrier Elimination (ESCE) method detailed in this paper proposes combining the two side lobes into a single lobe centered at the main frequency, thus gaining $2 \mathrm{~dB}$ more signal power as well as reducing the correlation requirements (signal's mixing and transforming operations) to the half; i.e. accelerating the acquisition process. HaLo-430 platform generated signals used for testing the MATLAB model of ESCE proves that we outperform three of the most used unambiguous methods.
\end{abstract}

Keywords-BOC modulation; Unambiguous Galileo signal Subcarrier elimination;

\section{Introduction}

The modulation of the Galileo's Binary Offset Carrier (BOC) signal multiplies the subcarrier frequency with the spreading codes and the carrier frequency. The subcarrier frequency separates the power spectrum into two symmetric side-lobes placed above and below the centre frequency. These side-lobes produce narrow multiple peaks when correlated with the receiver's generated BOC signal. The width of each peak is designed to be equivalent to one-third of the peak of the GPS signal in order to enhance the signal tracking accuracy as depicted in Figure 1. For a chip resolution of $\geq 0.5$ Chip, the cross-correlation function (CCF) becomes ambiguous because, with fewer generated peaks at

Ali Albu-Rghaif, Ihsan Lami, Maher Al-Aboodi

Department of Applied Computing

University of Buckingham

UK

first.last@buckingham.ac.uk

Patrick Van Torre, Hendrik Rogier

Department of Information Technology (INTEC-iMinds)

Ghent University

Belgium

first.last@UGent.be this resolution, the system may lock to the wrong peaks [1] Based on our literature survey, to overcome this ambiguous correlation problem, several solutions have been devised and detailed in section III, the most commonly used of these solutions recommend that:

1. The code's phase resolution must be divided by 3 to achieve the same correlation results as normally achieved with the GPS-BPSK signal [2], which means more processing time is required.

2. The BOC signal is processed as two BPSK signals, as illustrated in section III. This approach requires double processing, complicating the acquisition implementation.

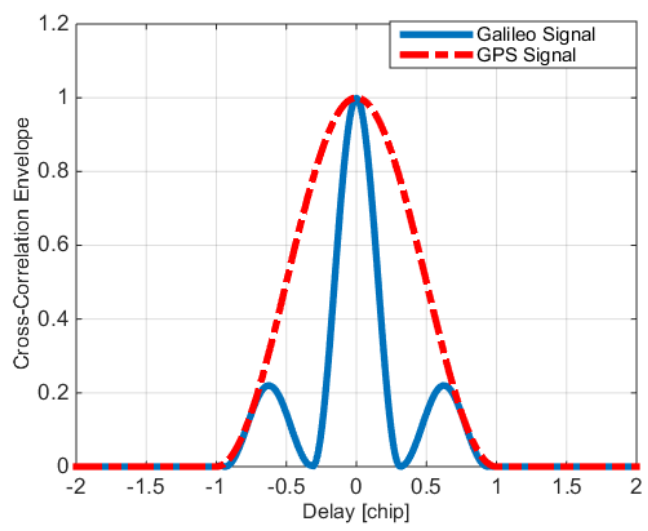

Figure 1. Cross correlation envelopes

This work proposes a new method to eliminate the "subcarrier frequency effect (SFE)" and to overcome the ambiguity condition as well as to enhance the acquisition process. As shown in Figure 4, the SFE elimination process is achieved by multiplying the received Galileo signals with either of the generated subcarrier signals (data or pilot) to convert the BOC modulation to a BPSK modulation before acquisition. As a result, the $\mathrm{CCF}$ effectively has only a single peak, similar to the GPS's CCF. This means that, existing GPS receivers would be able to acquire the Galileo signals without large overhead. The faster processing is achieved by only requiring a single channel for acquisition and also using a pure BPSK-PRN code. The details of the ESCE process are described in section IV.

The paper is organized as follows. Section II details the mathematical representation of the Galileo-OS signal. Section III summarises previously unambiguous acquisition methods. 
Section IV describes the implementation of our proposed method. Section V shows the results of the performance and the implementation requirements of our proposed method versus existing methods. Finally, the conclusions are listed in Section VI.

\section{The Mathematical Model of The Galileo-OS Signal}

The Galileo-E1-OS signal uses the Composite-BOC (CBOC) modulation, which means multi-level spreading symbols formed from the weighted sum of $\mathrm{BOC}(1,1)$ and $\operatorname{BOC}(6,1)$ as expressed in the following equations [3].

$$
g_{\text {Bос }(1,1)}= \begin{cases}\operatorname{sign}\left(\sin \left(\frac{2 \pi t}{T_{c}}\right)\right) & 0 \leq t \geq T_{c} \\ 0 & \text { elsewhere }\end{cases}
$$

and

$$
g_{\text {вос }(6,1)}= \begin{cases}\operatorname{sign}\left(\sin \left(\frac{12 \pi t}{T_{c}}\right)\right) & 0 \leq t \geq T_{c} \\ 0 & \text { elsewhere }\end{cases}
$$

where $\mathrm{g}_{\mathrm{BOC}(1,1)}$ represents the $\mathrm{BOC}(1,1)$ spreading symbols, $\mathrm{g}_{\mathrm{BOC}(6,1)}$ represents the $\mathrm{BOC}(6,1)$ spreading symbols, and $\mathrm{T}_{\mathrm{C}}$ is the code chip duration.

The $\mathrm{CBOC}(+)$ modulation is employed for the data channel $\left(\mathrm{S}_{\mathrm{b}}\right)$, while CBOC $(-)$ is used for the pilot channel $\left(\mathrm{S}_{\mathrm{c}}\right)$, as expressed below in $(1 \& 2)$.

$$
\begin{aligned}
& \mathrm{S}_{\mathrm{b}}=\alpha \mathrm{g}_{\mathrm{BOC}(1,1)}+\beta \mathrm{g}_{\mathrm{BOC}(6,1)} \\
& \mathrm{S}_{\mathrm{c}}=\alpha \mathrm{g}_{\mathrm{BOC}(1,1)}-\beta \mathrm{g}_{\mathrm{BOC}(6,1)}
\end{aligned}
$$

where $\alpha$ and $\beta$ are the power parameter to control the combined power of the data and pilot channels and are equal to $\sqrt{10 / 11}, \sqrt{1 / 11}$ respectively.

The mathematical representation of the transmitted CBOC signal is shown in equation (3) as described in the Galileo Signal-In-Space Interface Control Document (SIS-ICD) [4].

$$
S_{E 1}=\frac{1}{\sqrt{2}}\left[e_{E 1-B}(t) S_{b}-e_{E 1-C}(t) S_{c}\right] \cos \left(2 \pi f_{E 1} t\right)
$$

where $\mathrm{S}_{\mathrm{E} 1}$ represents the CBOC Galileo signal, the $\mathrm{e}_{\mathrm{E} 1-\mathrm{B}}$ and $\mathrm{e}_{\mathrm{E} 1-\mathrm{C}}$ are the binary signal components and $\mathrm{f}_{\mathrm{E} 1}$ is the carrier frequency.

Equation (3) clearly shows that the acquisition of Galileo signals can be obtained by either using single-channel acquisition (data or pilot signal) or dual-channel acquisition. For the sake of comparison, this work compares the performance and complexity with the most widely used solutions that are depending on single-channel acquisition methods.

\section{Literature survey of Unambiguous Methods}

We have studied the research literature on proposed methods for resolving the unambiguity issue of BOC signals, focusing on recent and most widely used methods. It is clear that the authors who formulated the BOC specification have actually described this ambiguity and pointed at possible solutions. The most obvious solution is to use chip resolution of less than 0.5 Chip.

One of the most common approaches is the dual sideband (DSB) method that was proposed to cope with the acquisition ambiguity from the BOC signal and to acquire the BOC signal as a BPSK signal. This method has been developed to acquire the GPS-M code signal, which is based on $\mathrm{BOC}(10,5)$ modulation [5]. Two filters have been used to filter the main side lobes (upper and lower side lobes) only, as illustrated in Figure 2. Thus, each lobe approximately represents a BPSK(5) signal, where the number five refers to the chipping rate $(5 * 1.023 \mathrm{MHz})$. The acquisition is accomplished through two distinct correlation channels for the upper and lower sidebands. Each correlation channel correlates the filtered signal with a filtered PRN-BOC modulated reference signal. The shape of the resulted $\mathrm{CCF}$ is approximately like the shape of the BPSK CCF.

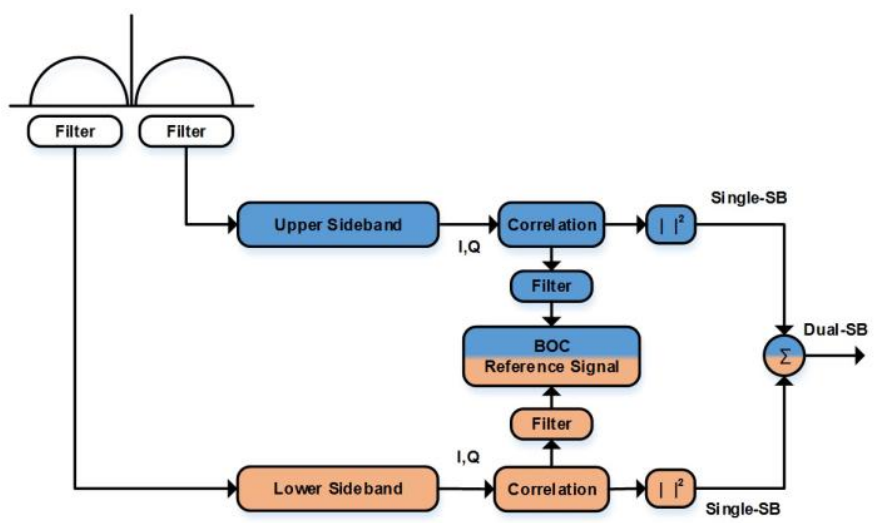

Figure 2. Dual sideband method

Similarly, BPSK-Like technique was designed to acquire the BOC signals. This technique is slightly different from the DSB method, where only a single filter is centered at the main carrier frequency, having a bandwidth that accommodates both of the main side lobes [6]. These main lobes are then shifted to the main centre frequency by the amount of the subcarrier frequency $\left(\mp_{\mathrm{sc}}\right)$. So, each sideband is correlated in parallel channels with the reference BPSK-modulated code as illustrated in Figure 3. This method is less complicated than the DSB method, where the reference code is represented by a BPSK-modulated code rather than a filtered BOC-modulated code. However, this approach will work with even BOC modulation orders only. Note that the use of a single sideband will introduce at least $3 \mathrm{~dB}$ degradation in the SNR of the received signal, but if these sidebands are combined then the loss can be partially compensated. 


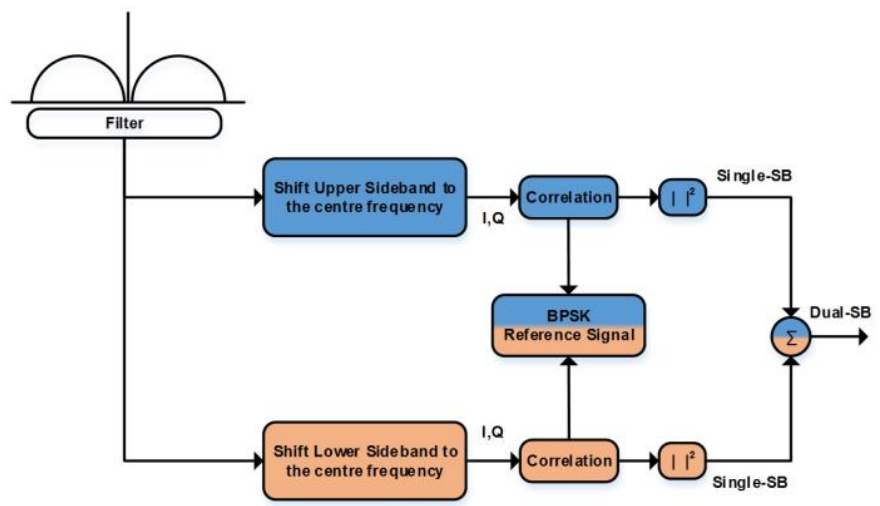

Figure 3. BPSK-Like method

Another approach is introduced to overcome the odd-order limitation of the BPSK-Like method, hence making the BPSK-Like method work for both even and odd BOC modulation orders $\left(\mathrm{N}_{\mathrm{BOC}}\right)$ [7]. This is achieved by determining the amount of shifting $\left(\delta * \mathrm{f}_{\mathrm{sc}}\right)$, where $\delta$ depends on the BOC modulation order and is equal to:

$$
\delta= \begin{cases}1 & \text { if } \mathrm{N}_{\mathrm{BOC}} \text { even, sin and cos }-\mathrm{BOC} \\ \frac{\mathrm{N}_{\mathrm{BOC}}-1}{\mathrm{~N}_{\mathrm{BOC}}} & \text { if } \mathrm{N}_{\mathrm{BOC}} \text { odd, } \sin \mathrm{BOC} \\ \frac{\mathrm{N}_{\mathrm{BOC}}+1}{\mathrm{~N}_{\mathrm{BOC}}} & \text { if } \mathrm{N}_{\mathrm{BOC}} \text { odd, } \cos \mathrm{BOC}\end{cases}
$$

To reduce the complexity of the above methods, three proposals have been designed for Low Complexity (LoCo) implementations [8]. The concept behind all of these three proposals is based on shifting the received BOC signal to the zero frequency and then generating a BPSK-PRN replica code. What distiguishes the different proposals is the way of using the filters. In the first proposal (modified DSB), the numbers of filters are reduced by generating BPSK-PRN code rather than generating filtered BOC-PRN code. The second proposal (modified BPSK-Like) introduces extra filters to overcome the BOC modulation order. The third proposal does not use any filtering. However, these proposals do not reach the same performance as the DSB method, albeit they reduce the implementation complexity.

Finally, a sub-carrier phase cancellation proposal is published that focuses on removing the subcarrier frequency of the received BOC signal in the acquisition process instead of in the pre-processing stage, as was the case for the DSB and BPSK-Like methods [9]. This is achieved by multiplying the BOC signal with the carrier frequency, and then both of the inphase and quadrature-phase correlation channels are multiplied by the local BOC signal-in-phase subcarrier and the local BOC signal-quadrature-phase subcarrier. Unambiguous CCF can be then obtained when all these in-phase and the quadrature-phase channels are combined, which is same as the CCF of the BPSK signal. In contrast to the above DSB, BPSK-Like and LoCo methods, this method does not depend on a filtering process to correlate the single or double sideband lobes, but it does cost more correlation channels, i.e. duplicates the numbers of required correlation channels (the in-phases \& quadrature-phases of the carrier frequency and the subcarrier frequencies). In addition, the performance of this method is not better than the performance of the BPSK-Like method.

\section{Iv. ESCE Method}

Our proposed ESCE method starts by multiplying the received signal by either the generated data channel's subcarrier (1) or by the generated pilot channel's subcarrier (2), as shown in Figure 4. Note that, the required code in this implementation is therefore either the primary code of the data channel if $\mathrm{CBOC}(+)$ is employed to eliminate the subcarrier frequency effect, or the primary code of the pilot channel if CBOC(-) is used.

The multiplication process is equivalent to shifting the two side lobes to the main frequency and simultaneously the powers of these lobes are also added together. Therefore, the resulting output signal a distinct BPSK signal. Our simulation shows that, this process produces a gain of $2 \mathrm{~dB}$ in the combined signal power compared to the actual received signal as shown in Figure 5. As a result of processing the BPSK signal, the envelope of the CCF is converted from multiple peaks to a single peak as shown in Figure 9.

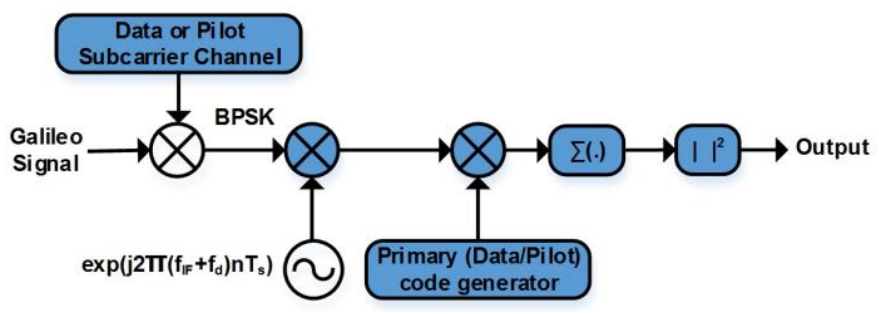

Figure 4. ESCE method

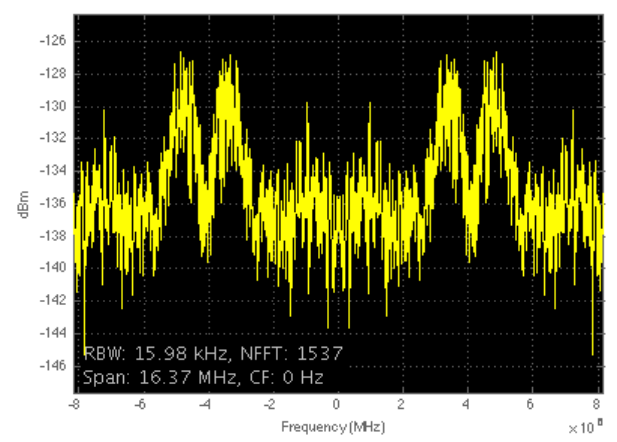

(a)

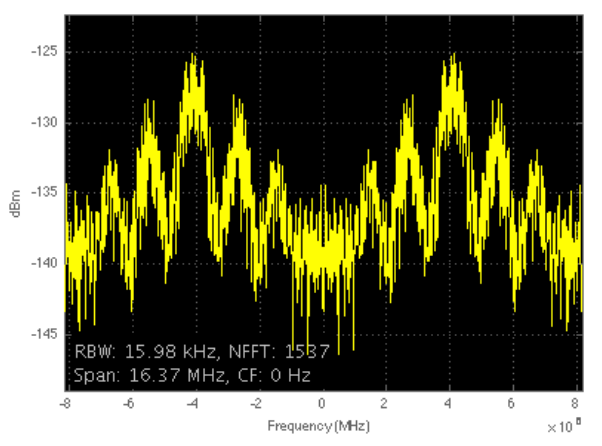

(b)

Figure 5. (a) Received Galileo signal, (b) our converted Galileo-BPSK signal using pilot subcarrier channel with $2 \mathrm{~dB}$ gain 
ESCE has two distinct advantages; the first is that the correlation requirements are reduced from (Carrier+Subcarrier+Code) to (Carrier+Code), and therefore existing GPS receivers can be readily used to acquire this converted Galileo signal without substantial overhead; the second is that the ESCE process can be implemented in the time-domain or in the frequency-domain.

\section{v. Experimental Results and Analysis}

In our experiments, the Galileo signals are transmitted and received in a real wireless communication channel by using the Signalion HaLo-430 platform [10], which is shown in Figure 6. This wireless testbed is fully controlled by MATLAB, and up to four signals, can be transmitted or received simultaneously. In each scenario we have used all four channels in the transmission and receiving platform to obtain various signal receptions in realistic environment.

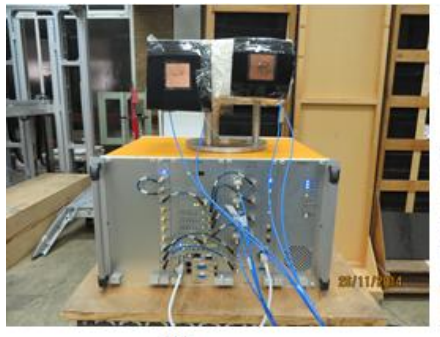

(a)

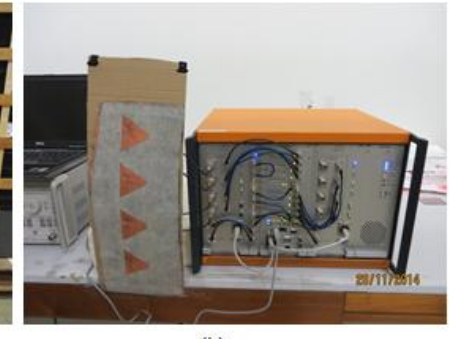

(b)
Figure 6. HaLo-430 (a) Transmitter (b) Receiver

As apart from the ESCE implementation, we have implemented three other unambiguous methods. These are the DSB method [5], the BPSK-Like method [6] and the LoCo method [8] as described in section III. This enabled us to compare ESCE to popular existing methods in equal conditions.

For processing time comparison, we performed Monte Carlo simulations with 100 runs to calculate the average time. In this comparison, all the methods are run with the same realistic signal processing scenario. As shown in Table I, the processing time achieved by ESCE is nearly half of the time required by the other methods. This proves that ESCE implementation is not only simple, but also faster.

TABLE I. PROCESSING TIME

\begin{tabular}{|l|l|}
\hline Method & Processing Time \\
\hline ESCE Method & $2.84 \mathrm{sec}$. \\
\hline DSB Method & $5.53 \mathrm{sec}$. \\
\hline BPSK-Like Method & $4.35 \mathrm{sec}$. \\
\hline LoCoMethod & $5.26 \mathrm{sec}$. \\
\hline
\end{tabular}

Figure 7 illustrates the computational complexity of ESCE compared with other methods. Again, ESCE is about $70 \%$ less computationally expensive.

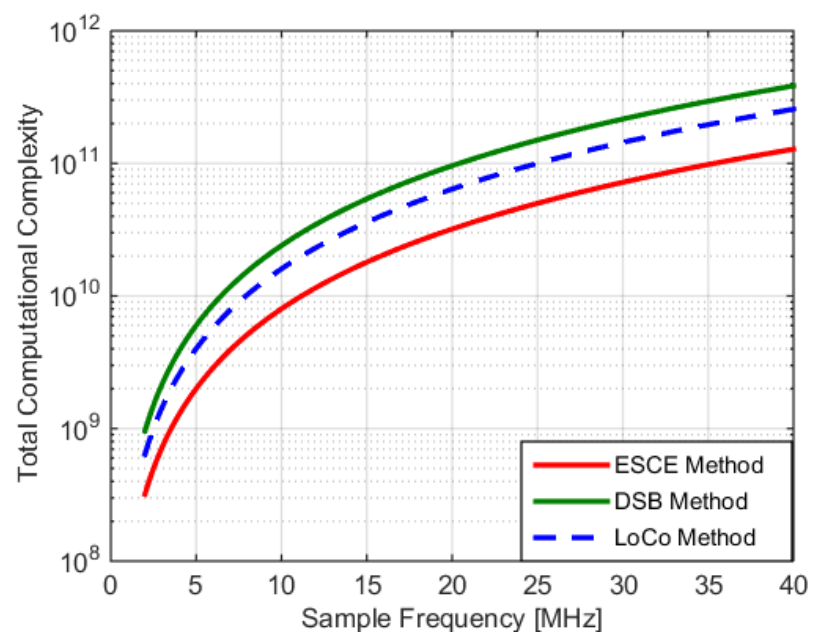

Figure 7. Total computational complexity

The performance comparison (probability of detection) between all methods is conducted by using an FFT-search algorithm with a PRN code length equal to 4092 Chips based on CBOC modulation and a Doppler frequency bin equal to $166.6 \mathrm{~Hz}$ (determined as $2 /(3 \mathrm{~T}) \mathrm{Hz}$, where $\mathrm{T}$ is the $4 \mathrm{~ms}$ search dwell time) [11]. Figure 8 illustrates the detection probabilities of ESCE compared with the BPSK-Like method and LoCo method. ESCE has better performance than BPSK-Like and LoCo methods by 1 and $2 \mathrm{~dB}$ respectively. This is due to the 2 $\mathrm{dB}$ gain obtained from ESCE conversion process.

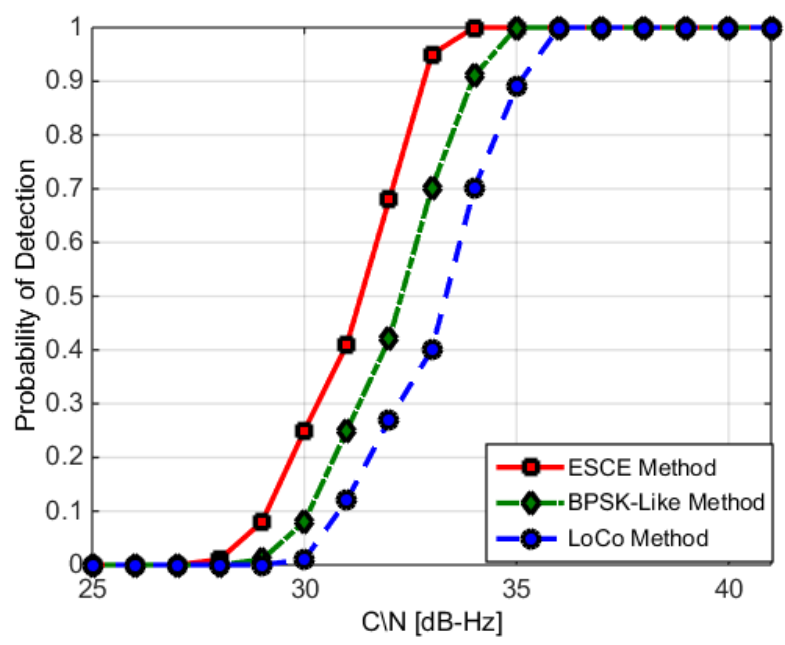

Figure 8. Probability of detection vs. ClN

Finally, Figure 9 shows the CCF of a time-domain implementation of ESCE having a single peak in comparison with an ambiguous method time-domain implementation which produces three narrow peaks. 

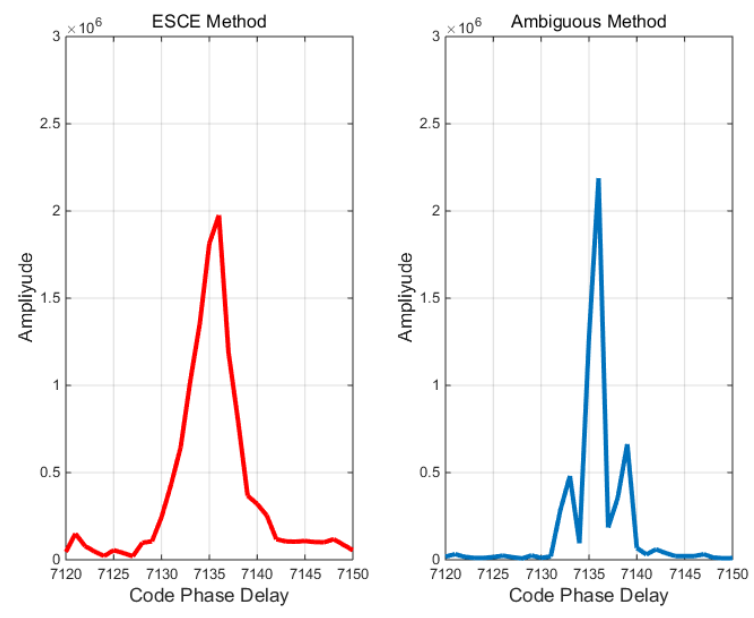

Figure 9. SV-2 Cross Correlation Function

\section{vi. Conclusion}

In this work, the authors propose ESCE, a new unambiguous acquisition method for the Galileo-El-OS signal. The implementation of ESCE has eliminated the subcarrier frequency effect and simplified the acquisition process.

The implementation requirements and detection performance of ESCE are analysed and compared to other widely used solutions, such as DSB, BPSK-Like and LoCo methods. The results showed the advantages ESCE in terms of reducing the complexity, improving the performance of the CBOC signal acquisition and accelerating the acquisition process. Our simulation shows that ESCE elimination of the subcarrier frequency effect offers around $2 \mathrm{~dB}$ gain to the received signal power.

In conclusion, our literature survey illustrates that unambiguous methods have been successful to acquire and track BOC signals. However, most of them suffer from having complicated implementations using double side band processing, or they suffer from a signal-to-noise deterioration, of around $3 \mathrm{~dB}$ power in case of single side-band processing. However, we believe that our solution of eliminating the subcarrier frequency produces better results than the previous work in terms of performance, saving processing time and implementation complexity. Furthermore, our acquisition method can be implemented in the time domain or in the frequency domain.

\section{Acknowledgment}

The researchers would like to thank the Iraqi Ministry of Higher Education for the PhD grant awarded to the first and third authors and also to the European COST Action IC1301 WiPE.

\section{References}

[1] L. Elena Simona, "Limited bandwidths and correlation ambiguities: Do they co-exist in Galileo receivers," Positioning, vol. 2011, 2011.

[2] W. De Wilde, J.-M. Sleewaegen, A. Simsky, J. Van Hees, C. Vandewiele, E. Peeters, J. Grauwen and F. Boon, "Fast signal acquisition technology for new GPS/Galileo receivers," Proc. of IEEE/ION PLANS'06, pp. 1074-1079, 2006.

[3] G. W. Hein, J.-A. Avila-Rodriguez, S. Wallner, A. R. Pratt, J. Owen, J.L. Issler, J. W. Betz, C. J. Hegarty, L. S. Lenahan, J. J. Rushanan and others, "MBOC: the new optimized spreading modulation recommended for GALILEO L1 OS and GPS L1C," in Proceedings of the IEEE/ION position, location, and navigation symposium, 2006.

[4] O. ICD, European GNSS (Galileo) open service. Signal in space. Interface control document. Issue 1.1, 2010.

[5] P. M. Fishman and J. W. Betz, "Predicting performance of direct acquisition for the M-code signal," in Proceedings of the 2000 National Technical Meeting of The Institute of Navigation, 2000.

[6] N. Martin, V. Leblond, G. Guillotel and V. Heiries, "BOC (x, y) signal acquisition techniques and performances," in Proceedings of the 16th International Technical Meeting of the Satellite Division of The Institute of Navigation (ION GPS/GNSS 2003).

[7] A. Burian, E. S. Lohan and M. Renfors, "BPSK-like methods for hybridsearch acquisition of Galileo signals," in Communications, 2006. ICC'06. IEEE International Conference on, 2006.

[8] E. S. Lohan, A. Burian and M. Renfors, "Low-complexity unambiguous acquisition methods for BOC-modulated CDMA signals," International Journal of Satellite Communications and Networking, vol. 26, no. 6, pp. 503-522, 2008.

[9] V. Heiries, D. Roviras, L. Ries and V. Calmettes, "Analysis of non ambiguous BOC signal acquisition performance Acquisition," 2004.

[10] [Online]. Available: http://www.telecomtest.com.au/datasheet/m011-v51_datasheet_halo430_2010.pdf.

[11] E. D. Kaplan and C. J. Hegarty, Understanding GPS: principles and applications, Artech House Publishers, 2006. 